Y. C. Minh and E. F. van Dishoeck, eds.

\title{
Organics in Space: From Interstellar Dust to Comets
}

\author{
J. Mayo Greenberg and Guillermo M. Muñoz Caro \\ Raymond and Beverly Sackler Laboratory for Astrophysics at Leiden \\ Observatory, University of Leiden, Postbus 9513, 2300 RA Leiden, The \\ Netherlands
}

\begin{abstract}
A cyclic evolutionary picture is presented which follows the sources and nature of organics from interstellar space to comets. The three major organic components discussed are the grain mantles, the carbonaceous particles responsible for the $216 \mathrm{~nm}$ hump in the extinction, and the large molecules/small particles polycyclic aromatic hydrocarbons (PAHs). The variability in the oxygen and hydrogen abundances relative to carbon is followed.
\end{abstract}

\section{Introduction}

There are several types of organics in interstellar space which have been catalogued. One of these is identified as the mantles on the $0.1 \mu \mathrm{m}$ interstellar grains primarily responsible for the visual extinction and polarization. This is observed spectroscopically via a $3.4 \mu \mathrm{m}$ absorption feature characteristic of $\mathrm{CH}$ stretches in $-\mathrm{CH}_{2}$ and $-\mathrm{CH}_{3}$ groups of aliphatic compounds. A second is associated with the $216 \mathrm{~nm}$ absorption hump and - for optical reasons - consists of particles at least ten times smaller. Finally, there is the source of the unidentified infrared emission features at $3.29 \mu \mathrm{m}, 6.2 \mu \mathrm{m}, 7.7 \mu \mathrm{m}, 8.85 \mu \mathrm{m}$, and $11.3 \mu \mathrm{m}$ which has been strongly indicated to be in the form of large polycyclic aromatic hydrocarbon (PAH) molecules (Léger \& Puget 1984; Allamandola et al. 1985). Evidence for organics in circumstellar regions and in meteorites and comets is abundant. A fundamental question is to what extent these are related and, if so, what is the evolutionary sequence which connects them. What are the sources and sinks of the various components? These questions of evolution and sources and sinks are ultimately coupled and will be discussed in the following sections.

\section{Grain Mantles: Variations in the $3.4 \mu \mathrm{m}$ Feature}

\subsection{The $3.4 \mu \mathrm{m}$ feature}

The scenario which predicts the organic mantles starts with small silicate particles being produced and blown out of cool evolved stars. Since silicates alone are not capable of providing the observed amount of extinction (Aitken et al. 1989; Henning \& Stognienko 1993) one requires a contribution by carbonaceous components. Oxygen-rich ones are too volatile. Carbonaceous components separated from the silicates do not appear to satisfy the known constraints but as 
mantles on the silicates they may be shown to satisfy all the major constraints as well as, or better than, any other grain model ( $\mathrm{Li} \&$ Greenberg 1998). The production of such mantles is likely to be the result of ultraviolet processing of the icy mantles at some stage in the molecular cloud phase. As a representation of such processing, laboratory experiments have been performed by irradiating various ice mixtures deposited at temperatures as low as $10 \mathrm{~K}$ which when warmed up to room temperature leave a complex organic residue (Greenberg et al. 1972; Hagen, Allamandola, \& Greenberg 1979; Briggs et al. 1992). This material is presumed to be similar to the first generation of organics before the dust emerges into the diffuse cloud phase. Such organics may, and probably do, resemble some of the organics to be found in comets which have formed out of the last contraction of a molecular cloud. However, those dust grains which are cycled back to the diffuse cloud will lose all their volatiles readily (Draine \& Salpeter 1979; Greenberg 1982a,b) leaving only an organic residue. This organic is then subjected to the intense ultraviolet flux of the diffuse cloud medium leaving an organic which is carbonized in the sense that it consists of first generation organics which have been depleted in oxygen and hydrogen.

A test of this organic component has been obtained by exposing first generation organic residues to the solar ultraviolet radiation for the equivalent of $10^{6}$ years of radiation in the diffuse cloud medium, and comparing the infrared absorption feature at $3.4 \mu \mathrm{m}$ with that observed to the galactic center. The comparison is remarkably good (see Figure 1). An alternative to this analog has been offered by the creation of hydrogenated amorphous carbon (HAC) and carbon grains (ACARL), see Mennella et al. (1999). The $3.4 \mu \mathrm{m}$ absorption is similar to that of the 2nd generation organics but it is not quite as good compared with the observations. The observed absorption towards the Galactic Center at $2870 \mathrm{~cm}^{-1}$ appears to be a bit larger than that of the carbon grains (ACARL), which might indicate that the aliphatics of carbon grains are slightly longer. A further disadvantage of the $\mathrm{HAC}$ as grain mantles is that it requires relatively more carbon so that the cosmic abundance constraint is violated (see section 6).

The correlation of the $3.4 \mu \mathrm{m}$ feature with silicate absorption has provided a strong confirmation of the core-mantle construction (Pendleton et al. 1994). There are however two arguments against this construction: (i) no $3.4 \mu \mathrm{m}$ features observed in molecular clouds (Allamandola et al. 1992), (ii) no observed polarization in the $3.4 \mu \mathrm{m}$ feature in IRS 7 (Adamson et al. 1999). The former objection may be addressed by considering what happens to the organic mantle when it accretes ices and is simultaneously subjected to ultraviolet radiation. Such experiments have been performed in the Leiden laboratory with the result that a thin ice mantle allows for the dehydrogenation of the underlying organic refractory layer while inhibiting the rehydrogenation by colliding hydrogen atoms (Muñoz Caro et al. 2000). The second objection has no final answer. If the $3.4 \mu \mathrm{m}$ feature is seen in absorption and if the silicate feature is polarized one should also see polarization in the $3.4 \mu \mathrm{m}$ feature just as the polarization of the ice features goes along with the silicate polarization in the Orion BN object (Gillett et al. 1975; Capps et al. 1978). Without direct evidence for silicate polarization in IRS 7 the absence of $3.4 \mu \mathrm{m}$ polarization is not conclusive evidence against grain mantles. Furthermore, if the $3.4 \mu \mathrm{m}$ feature is not due to the mantle and if it is due to another grain component, the question 


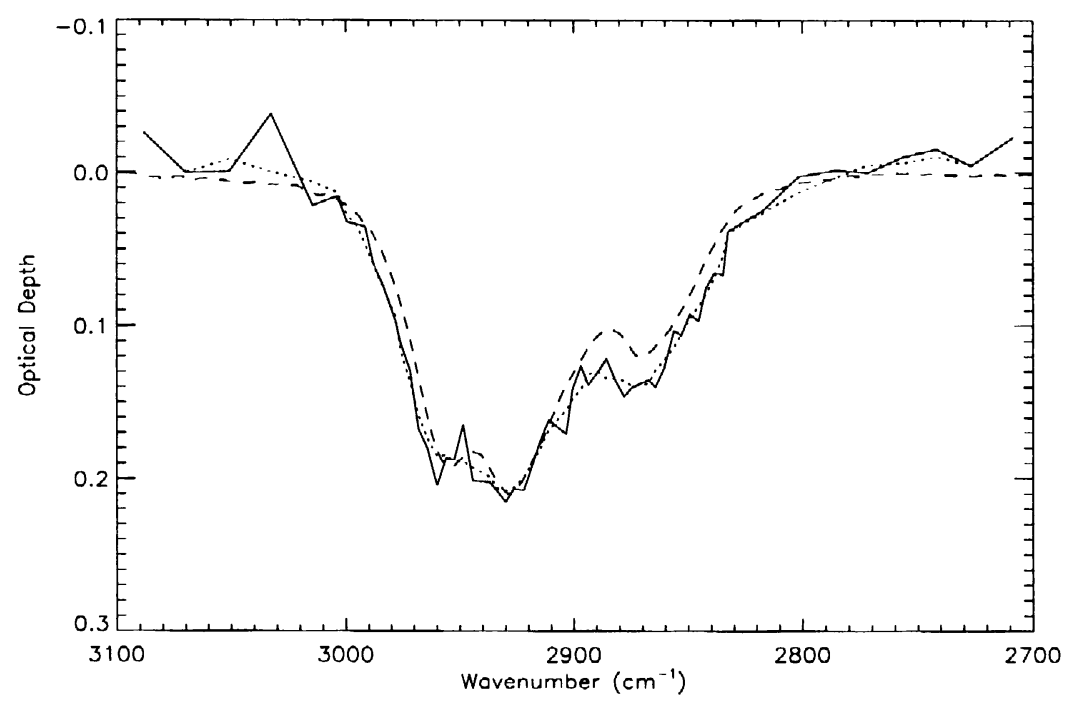

Figure 1. Comparison of the $3.4 \mu \mathrm{m}$ feature of IRS $6 \mathrm{E}$ (solid line), towards the Galactic center, with EURECA A sample (dots) and an ACARL sample (dashed line).

is which one? The only possible candidate would appear to be the small isolated carbonaceous particles which are those which provide the $216 \mathrm{~nm}$ absorption. An objection to this hypothesis as a general condition is that the hump particles almost certainly consist of dehydrogenated highly conjugated carbon; i.e., as in PAHs and these possess extremely weak $3.4 \mu \mathrm{m}$ absorption (see section 6).

The exclusion of organic mantles would make it difficult to provide the excellent fit to the silicate band in the Orion BN polarization because it appears to be needed to provide the correct position and width (Greenberg \& $\mathrm{Li} 1996$ ). A source of the $3.4 \mu \mathrm{m}$ feature has been suggested as coming from circumstellar regions rather than from mantles (Chiar et al. 1998). When such particles (presumed very small) emerge into space, they are subject to destruction (see section 5.1) and dehydrogenation (see section 3) which make them relatively weak contenders for the interstellar $3.4 \mu \mathrm{m}$ absorption.

\section{Hump Particles}

The existence of a separate population of small carbonaceous particles responsible for the $216 \mathrm{~nm}$ hump dates back to 1965 when graphite was suggested as the carbonaceous material. That the particles have to be small is convincingly argued on their optical properties alone (see Chlewicki \& Greenberg 1990). They are pure absorbers at $216 \mathrm{~nm}$ and the only particles which can be pure absorbers at this wavelength are those which have $2 \pi a / \lambda<0.3$ which implies 
$a<\lambda / 2 \pi \simeq 0.01 \mu \mathrm{m}$. This puts them out of the class of the $0.1 \mu \mathrm{m}$ particles responsible for the visual extinction. It also puts them outside of the class of particles which can accrete mantles for several reasons, one of which is the temperature fluctuations induced by UV photon absorption and the other is the energy released by molecule formation (Greenberg 1980). That the particles are not graphite can be deduced from the fact that the shape of the hump requires some particles to be large enough to contribute to the FUV extinction and this is contradicted by the lack of the correlation between the hump strength and the FUV (Greenberg \& Chlewicki 1990). This leaves as a candidate some, as yet, not fully identified form of carbon. One thing is clear and that is that size restriction alone is not sufficient to define the band shape. Most of the carbonaceous candidates so far suggested have been derived from laboratory samples of carbonaceous materials. The general problem is that while there are many materials which have a strong absorption 'in the neighborhood' of $216 \mathrm{~nm}$ most absorb at a somewhat longer wavelength and furthermore give too large a contribution to the visual extinction which can be shown to be inconsistent with the observational constraint provided by the ratio of visual polarization to extinction because the hump particles are not aligned. Some progress towards understanding what kinds of carbonaceous materials may evolve in space to produce the $216 \mathrm{~nm}$ hump has been made by Mennella et al. (1998) by showing that the narrowing of the measured absorption hump by hydrogenated amorphous carbon occurs as a result of radiation which dehydrogenates the carbon. Thus a strongly dehydrogenated organic material is suggested.

\section{PAHs}

We shall not dwell too much on these fascinating PAH molecular components of the ISM. For details we refer to the article by Tielens, this volume. The main point we wish to make here is that of the question of where they come from. It is reasonably certain that about $20 \%$ of all available carbon is taken up by these PAHs. Many theoretical and experimental arguments have been suggested for the destruction of PAHs (see section 5). If one accepts that the mean lifetime of a PAH is the same as that of all other grain components, namely, of the order of $5 \times 10^{8}$ years, it turns out that taking all possible known circumstellar sources of these molecules can account for at most $10 \%$ of those observed. Consequently one is forced to consider the interstellar medium itself as the prime source of those observed. The processing of carbon-bearing molecules starting with carbon atoms leading to $\mathrm{CH}_{4}, \mathrm{CO}, \mathrm{CO}_{2}, \mathrm{CH}_{3} \mathrm{OH}$ on interstellar dust is well established. The further processing of these ices leading to complex organics has been demonstrated by laboratory analog experiments. In section 2 the subsequent processing of these first generation organics has been shown to lead to a new form of organics. It has now been shown that these UV processed organics are very rich in PAHs (Greenberg et al. 2000), much richer than the first generation organics, and that the $3.4 \mu \mathrm{m}$ feature characterizes only about $20 \%$ of the grain mantles, the major fraction consisting of highly conjugated carbon molecules. Thus one has a potential source of PAHs adequate to account for the observed abundance if one can release them from the grain mantles. This will be suggested in the next section. 


\section{Sources and Sinks Evolution}

\subsection{Formation and destruction}

The major considerations in our understanding of the evolution of the solid particles in the ISM are the sources and sinks. Circumstellar regions and supernovae are the most serious contenders as sources. The rates of production of carbonaceous solids (SC) including large molecules like PAHs by all known, or estimated, stars is $0.003 \mathrm{M}_{\odot} \mathrm{yr}^{-1} \leq \dot{M}_{\mathrm{SC}}^{C S} \leq 0.01 \mathrm{M}_{\odot} \mathrm{yr}^{-1}$ (Gehrz 1989). The rate of production of organic refractories in the molecular cloud phase is $\dot{M}_{\mathrm{SC}}^{I S M} \simeq 0.01 \mathrm{M}_{\odot} \mathrm{yr}^{-1}$ (Greenberg 1986). The rate of destruction of carbonaceous solids (and large PAHs) is $\dot{M}_{\mathrm{SC}}^{I S M}=-0.1 \mathrm{M}_{\odot} \mathrm{yr}^{-1}$ if we assume that the mass of carbonaceous material is equivalent to the mass of silicates and about equal destruction rates (Draine \& Salpeter 1979; Greenberg 1982a,b). This is at least 10 times larger than the rate of production for all sources excluding the ISM. Given the fact that the carbon depletion by the hump particles and the PAHs together as deduced from their contribution to the interstellar extinction is about $40 \%$ ( $\mathrm{Li} \&$ Greenberg 1998), the source of these components must be found in the ISM.

\subsection{The cyclic evolutionary picture}

Although the rates of production and destruction are generally stated as averages it is well recognized that the processes occur in different regions. In particular, the destruction of most solid particles occurs generally in the low density regions where they are unshielded from shocks induced by supernovae explosions. A possible exception to this is the apparent disappearance and 'destruction' of PAHs which can occur in molecular clouds (Mendoza Gómez et al. 1995). While destruction time scales are $\sim 5 \times 10^{8}$ years, the residence time for interstellar dust in the diffuse and molecular cloud phases is significantly less, being about $5 \times 10^{7}$ years in each. We thus envisage dust components passing through one complete cycle each $10^{8}$ years. In order to maintain a mean steady state dust composition it is necessary to resupply the components destroyed in one phase by those produced in the other phase. While it has been demonstrated by laboratory analog experiments that the organic refractories are produced adequately in the molecular cloud phase to compensate for their destruction ('erosion') in the diffuse cloud (DC) phase, the precise physical mechanism for the erosion process is not well established. Is it by grain mantle shattering in grain-grain collisions or by shock induced sputtering or both? A way to account for the abundance of hump particles and of PAHs is to accept the possibility that they are products of the partial destruction of organic grain mantles in the diffuse cloud phase. If small pieces of the order of or less than $0.01 \mu \mathrm{m}$ (the mean thickness of organic mantles is $\approx 0.015 \mu \mathrm{m}$ as discussed in the next section) are broken off as a result of grain-grain collisions, these small particles are then subjected to extreme ultraviolet processing (Jenniskens \& Greenberg 1993). Collision frequencies of bondbreaking photons as compared with hydrogen atoms in the diffuse medium are $\Phi_{h \nu} / n_{\mathrm{H}} V_{\mathrm{H}} \simeq 10^{8} / 10^{6}=10^{2}$ (where $\Phi_{h \nu}=$ photon flux at $\mathrm{h} \nu>6 \mathrm{eV}, n_{\mathrm{H}}=$ number of hydrogen atoms, $V_{\mathrm{H}}=$ hydrogen speed) so that these small particles are efficiently dehydrogenated. This process appears to ultimately produce a kind of hydrocarbon which can reproduce the $216 \mathrm{~nm}$ hump 


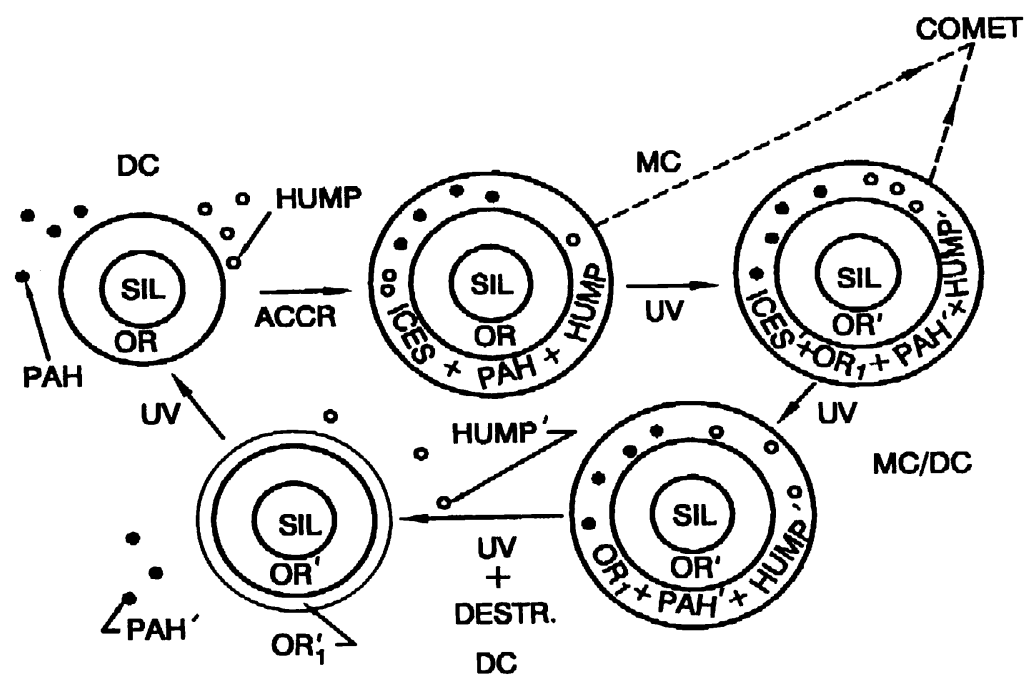

Figure 2. Scheme of the evolution of the organic component of interstellar dust. $\mathrm{DC}=$ diffuse cloud phase $\mathrm{MC}=$ molecular cloud phase; $\mathrm{OR}=$ average diffuse cloud organic refractory mantle material; $\mathrm{OR}_{1}=$ first generation organics; components with a superscript $\left({ }^{\prime}\right)$ are modified forms. See text for additional descriptions.

(Mennella et al. 1998). Perhaps a process of more serious erosion of the protohump particles in combination with direct erosion of the organic mantles leads to the formation of another form of highly dehydrogenated hydrocarbons in the form of PAHs and proto-PAHs and other highly conjugated large molecules. The general picture of the cyclic evolution is shown in Figure 2 which schematically shows the cycle from diffuse cloud dust to molecular cloud and back to diffuse cloud dust. The existence of breaks in the cycle as exemplified by the formation of comets out of the dust in pre-stellar clouds is also indicated and its consequences will be discussed in section 7 .

\section{The Unified Dust Model}

Up to this point we have considered primarily the physical and spectral evolutionary properties of dust. In addition to these clues to dust composition there are the classical ones based on their continuum optical properties of extinction, polarization and albedo. The major mass of the dust is in the core-mantle component which provides the linear polarization produced by aligned particles.

In the unified dust model there are large silicate core-organic refractory mantle dust particles; very small carbonaceous particles responsible for the 
hump extinction; and PAHs responsible for the FUV extinction. The model results are in good agreement with such observational constraints as the average interstellar extinction curve, the polarization law, the ratio of visual polarization to extinction $(P / A)_{v}$, the scattering properties (albedos), and the excess NIR polarization over the extrapolation of the Serkowski law. The $(P / A)_{v}$ constraint imposed on other dust models (e.g. the silicate/graphite model, the composite dust model) leads to either a too low $(P / A)_{v}$ value (the silicate/graphite model) or instability of particle structure (the composite model). The cosmic abundance constraint, in particular the evidence for a lower oxygen abundance in the interstellar medium than in the solar system and the possible interstellar $\mathrm{C} / \mathrm{O}$ ratios, are discussed extensively. The relative atomic composition of the mantle is assumed to be $\mathrm{C}: \mathrm{O}: \mathrm{N}: \mathrm{H}=1.0: 0.2: 0.04: 1$ which is based on the measurements of laboratory organics and the Comet Halley mass spectra (Greenberg et al. 1992; Schutte 1988; Kissel \& Krueger 1987). With $2.3 \mathrm{~g} \mathrm{~cm}^{-3}$ and $2.4 \times 10^{-7}$ $\mathrm{g} \mathrm{cm}^{-2}$ as the mass density of the hump particles and the PAHs (assumed to be essentially 2 dimensional) respectively and from the number densities $n_{\mathrm{cm}}$, $n_{\text {hump }}, n_{\text {PAH }}$ and the sizes determined for fitting the interstellar extinction curve and the polarization law as well as the chemical compositions, we can obtain $(x / \mathrm{H})_{d}^{\text {mod }}$, the amount of each species $x$ locked in the dust particles. Although our model requires a bit more carbon than the reference abundances, it is within the limit of an acceptable range and is a significant improvement over other models. All the other major reference abundance (cosmic - non-dust) constraints on $\mathrm{O}, \mathrm{N}, \mathrm{Si}, \mathrm{Mg}, \mathrm{Fe}$ are well satisfied. Note that if the $\mathrm{O}: \mathrm{C}$ ratio in the grain mantle is reduced to zero (HAC) the carbon dust requirement is $20 \%$ higher and therefore more difficult to satisfy.

In conclusion, to get back to the apparent absence of a $3.4 \mu \mathrm{m}$ polarization, we note that, if both the hump excess polarization and the hump extinction at $216 \mathrm{~nm}$ are really produced by the same carrier, the degree of alignment and/or polarizability of such particles should be very small. For example, along the line of sight to $\mathrm{HD} 197770$, the ratio of excess polarization to the hump extinction is $P_{\mathrm{H}} / \mathrm{A}_{\mathrm{H}} \simeq 0.002$ (Clayton et al. 1992; Anderson et al. 1996). However, the polarization to extinction ratio in the visual is $P_{v} / A_{v} \simeq 0.025$, so that $\left(P_{\mathrm{H}} / \mathrm{A}_{\mathrm{H}}\right) /\left(P_{v} / A_{v}\right.$ is only $\approx 0.09$ and the contribution of the hump particles to the polarization in the visual (or the $3.4 \mu \mathrm{m}$ ) would indeed be much smaller than that by the core-mantle particles. Suppose we attribute the $3.4 \mu \mathrm{m}$ absorption feature to a part of the hump particle population rather than exclusively to the mantles. We then are facing the problem that such particles would contain too much hydrogen to produce the right position and shape of the hump absorption (Mennella et al. 1998).

We conclude that the observed lack of polarization at $3.4 \mu \mathrm{m}$ cannot readily be attributed to the $3.4 \mu \mathrm{m}$ absorption feature by the hump particles even though they are at best poorly aligned.

\section{Comet Organics}

There are two types of observations on which one bases the presence of and amount of organics in comets. The direct one was that provided by the mass spectra of comet Halley dust obtained by time-of-flight mass spectrometers on 
the Giotto and Vega satellites. The indirect ones are: (1) the presence of distributed molecules such as $\mathrm{CO}$ and $\mathrm{CN}$ in the coma presumed to be produced from large organic parent molecules and (2) the strength of the $10.0 \mu \mathrm{m}$ emission features in heated comet dust.

\subsection{Comet Halley mass spectra}

In March of 1986 several spacecrafts went by Halley's comet. Time-of-flight mass spectrometers carried aboard provided in situ measurements of the chemical composition of the dust particles emitted from the nucleus of the comet (Kissel et al. 1986a, b). The variation in the composition from one grain to another was found to be surprisingly large. Although the ratio of rock forming elements to those forming organic compounds varied enormously, there was no hint of a separate occurrence of one component without the other. This intimate mixture of rocky and organic solids as well as the wide variation of chemical composition points to the fact that this material is indeed left over from the formation of the solar system. The first result that became available after the fly-bys was the elemental analysis of cometary dust particles. Due to the high impact speeds, singly charged atomic ions formed $99.9 \%$ of the mass spectra. Within the accuracy of the ion yields (factor of 2) the major rock forming elements were found to occur at relative abundances similar to $\mathrm{CI}$ chondrites or solar composition. The very volatile elements $\mathrm{H}, \mathrm{C}, \mathrm{N}$, and $\mathrm{O}$, however, were more abundant in the cometary dust relative to the CI chondrites, yet still depleted relative to the solar photosphere. Further analysis of the data showed that the organic molecules had, on the average, a higher initial energy than the silicate ions which led Kissel \& Krueger (1987) to infer a core-mantle structure of the dust particles. Thus according to Jessberger \& Kissel (1991) "The existence of the previously postulated (Greenberg 1982a) core-mantle grains seems to be substantiated by data." An $\mathrm{O}: \mathrm{C}=0.6: 1$ ratio deduced from the overall comet Halley organics is much higher than deduced from the Galactic center absorption spectra but is consistent with a mixture of first generation laboratory organics with space irradiated organics as implied by the lab residues returned after being exposed to the Sun for 6 months (Greenberg et al. 1995). If we assume that the comet material contains the solar system abundance of the elements and if we assume that the $\mathrm{Si}, \mathrm{Mg}$ and $\mathrm{Fe}$ are totally bound up in the silicate cores we may derive a mantle to core mass ratio. This turns out to be (Greenberg 1998) $m_{\mathrm{OR}} / m_{\text {sil }} \approx 1$ which is in agreement with the value obtained by the Giotto/Vega space mission to comet Halley (Kissel \& Krueger 1987) as well as with the Orion BN polarization requirement if $\rho_{\mathrm{OR}} \simeq 1 / 2 \rho_{\text {sil }}$ (Greenberg \& Li 1996).

In addition to the "large" organic mantles there have been observed very small particles, tentatively identified as carbonaceous, by Utterback \& Kissel (1990) in the comet Halley dust spectra. And finally the PAH molecule phenanthrene has been identified as evidence for previously produced interstellar PAHs (Moreels et al. 1994).

The amount of $\mathrm{CO}$ and $\mathrm{CN}$ in the coma of comet Halley and spatial distribution indicates long time surviving parent molecules; i.e., they can be attributed to the heating of comet dust of a highly porous nature. The porosity is needed to provide a high temperature for relatively large mass particles as well as the large surface area for the evaporation of the small molecules. Furthermore, the 
detection of the distributed CO molecule in the comet Halley coma provides an important clue to the presence of comet organics and their oxygen abundance. Eberhardt et al. (1987) found that rather than falling off as $1 / R$, the CO intensity showed an approximately exponential growth up to about $20,000 \mathrm{~km}$. This was explained in terms of an extended source attributable to the evaporation of that organic fraction of comet dust which is rich in oxygen (Greenberg \& Hage 1990; Greenberg \& Li 1998).

\subsection{Heated dust grains}

A major contribution to comet science was made by the discovery on the Giotto and Vega satellite of a large number of small particles among the comet dust (McDonnell et al. 1986). However, even though there was a surprinsingly large number of particles small enough to emit spectrally at the $9.7 \mu \mathrm{m}$ characteristic of silicate there were not enough to provide the observed amount of silicate emission. It turns out that not only for comet Halley but also for Hale-Bopp and other comets, the requirement is that even particles with masses as high as $10^{-10} \mathrm{~g}$ must be able to emit spectrally. The two basic requirements then are that the particles be very fluffy aggregates of $0.1 \mu \mathrm{m}$ size particles and that there be a material present which absorbs well at visual wavelengths. The latter is achieved by mantleing the silicates with organics. The required degree of porosity (fluffiness of the aggregates) is defined by the fact that for a given mass the more porous the aggregate, the more it acts like a sum of small particles rather than a compact particle of that mass, so that a larger fraction of the observed mass distribution provides silicate emission as if by submicron particles. For example, the temperature of a $10^{-11} \mathrm{~g}$ compact grain made of silicate coreorganic refractory mantle materials with a mass ratio of the mantle to the core of $1 / 2$ at $1 \mathrm{AU}$ is $\approx 416 \mathrm{~K}$, while at a porosity of $P=0.975$ it is $\approx 706 \mathrm{~K}$ (we assume here that about $1 / 2$ of the organics are relatively volatile). The temperature for a pure silicate grain is $\approx 340 \mathrm{~K}$ (if compact) and $\approx 451 \mathrm{~K}$ (if $P=0.975$ ), respectively (Greenberg \& $\mathrm{Li} \mathrm{1998).} \mathrm{The} \mathrm{point} \mathrm{here} \mathrm{is} \mathrm{that} \mathrm{in} \mathrm{no} \mathrm{way} \mathrm{will} \mathrm{the}$ emissivity at $10 \mu \mathrm{m}$ be significantly above the continuum without both high porosity and organics for heating effectively.

\section{Summary}

While not all stages in the evolution of interstellar organics are completely clear, the trend starting with the production of oxygen- and hydrogen-rich organics as grain mantles in molecular clouds seems to provide a basic source. The photoprocessing of organics in the diffuse cloud phase produces a new variety of organics which are highly conjugated. A mechanism for producing the hump particles and the free flying PAHs is suggested starting with the shattering of grain mantles by grain-grain collisions and subsequent photoprocessing of the debris. The destruction and photoprocessing of circumstellar organics after arrival in interstellar space appears to put severe limits of $\leq 10 \%$ on their abundance as dust components. It is shown that some of the well observed properties of comets can self-consistently be described in terms of the core-mantle particles with the addition of the interstellar hump and PAH particles as trapped in the icy mantles of the final collapsing cloud phase. 
Acknowledgments. We thank W. Schutte for fruitful discussions. We are grateful to V. Mennella for kindly supplying us data on the $3.4 \mu \mathrm{m}$ feature of carbon grains. One of us (GMMC) wishes to thank the Max Planck Institute for Aeronomy for a fellowship, and in particular the COSAC-Rosetta team.

\section{References}

Adamson, A.J., Whittet, D.C.B., Chrysostomou, et al. 1999, ApJ, 512, 224

Aitken, D.K., Smith, C.H., \& Roche, P.F. 1989, MNRAS, 236, 919

Allamandola, L.J., Tielens, A.G.G.M., \& Barker, J.R. 1985, ApJ, 290, L25 1989, ApJS, 71, 733

Allamandola, L.J., Sandford, S.A., Tielens, A.G.G.M, \& Herbst, T.M. 1992, ApJ, 399, 134

Anderson, C.M., et al. 1996, AJ, 112, 2726

Briggs, R., Ertem, G., Ferris, J.P., Greenberg, J.M., McCain, P.J., Mendoza Gómez, C.X., \& Schutte, W. 1992, Origins of Life and Evolution of the Biosphere, 22, 287

Capps, R.W., Gillett, F.C. \& Knacke, R.F. 1978, ApJ, 226, 863

Chiar, J., Pendleton, Y.J., Geballe, T.R., et al. 1998, ApJ, 507, 281

Chlewicki, G. \& Greenberg, J.M. 1990, ApJ, 365, 230

Clayton, G.C., et al. 1992, ApJ, 385, L53

Draine, B.T. \& Salpeter, E.E. 1979, ApJ, 231, 438

Eberhardt, P., et al. 1987, A\&A, 187, 481

Gehrz, R.D. 1989, in Interstellar Dust, eds. L.J. Allamandola \& A.G.G.M. Tielens (Dordrecht: Kluwer), 445

Gillett, F.C., Forrest, W.J., Merrill, K.M., Capps, R.W., \& Soifer, B.T. 1975, ApJ, 200, 609

Greenberg, J.M. 1980, Université de Liège, 555

1982a, in Comets, ed. L.L. Wilkening (Univ. of Arizona press), 131

1982b, in Submillimetre Wave Astronomy, eds. J.E. Beckman \& J.P. Phillips (Cambridge University Press), 261

- 1986, in Light on Dark Matter (Proc. IRAS Symp., Noordwijk 10-14 June 1985), ed. F.P. Israel (Dordrecht: Reidel), 177 1998, A\&A, 330, 375

Greenberg, J.M. \& Chlewicki, G. 1983, ApJ, 272, 563

Greenberg, J.M., Gillette, J.S., Muñoz Caro, G.M., et al. 2000, ApJL, in press

Greenberg, J.M. \& Hage, J.I. 1990, ApJ, 361, 260

Greenberg, J.M. \& Li, A. 1996, A\&A, 309, 258 1998, A\&A, 332, 374

Greenberg, J.M., Li, A., Mendoza Gómez, C.X., Schutte, W.A., Gerakines, P.A., \& de Groot, M. 1995, ApJ, 455, L177

Greenberg, J.M., Mendoza Gómez, C.X., de Groot, M.S., \& Breukers, R. 1992, in Dust and Chemistry in Astronomy, eds. T.J. Millar \& D.A. Williams (IOP publ.), 265

Greenberg, J.M., Schutte, W.A., \& Li, A. 1999. Adv. Space Res., 23, no. 2, 289

Greenberg, J.M., Yencha, A.J., Corbett, J.W., \& Frisch, H.L. 1972, Mém. Soc.

Roy. Sci. Liège, 6e série, tome III, 425

Hagen, W., Allamandola, L.J., \& Greenberg, J.M. 1979, Ap\&SS, 65, 215

Henning, Th. \& Stognienko, R. 1993, A\&A, 280, 609 
Jenniskens, P. \& Greenberg, J.M. 1993, A\&A, 274, 439

Jessberger, E.K. \& Kissel, J. 1991, in Comets in the Post-Halley Era, eds. R.L. Newburn, et al. (Dordrecht: Kluwer), 1075

Kissel, J., Brownlee, D.E., Büchler, K., et al. 1986a, Nature, 321, 336

Kissel, J., Sagdeev, R.Z., Bertaux, J.L., et al. 1986b, Nature, 321, 280

Kissel, J. \& Krueger, F.R. 1987, Nature, 326, 755

Léger, A. \& Puget, J.L. 1984, A\&A, 137, L5

Li, A. \& Greenberg, J.M. 1997, A\&A, 323, 566 1998, ApJ, 498, L83

Mc Donnell, J.A.M., et al. 1986, Nature, 321, 338

Mendoza-Gómez, C.X., de Groot, M.S., \& Greenberg, J.M. 1995, A\&A, 295, 479

Mennella, V., Brucato, J.R., \& Colangeli, L. 1999, ApJ, 524, L71

Mennella, V., Colangeli, L., Bussoletti, E., et al. 1998, ApJ, 507, L177

Moreels G., Clairemidi J., Hermine P., Brechignac P., \& Rousselot P. 1994, A\&A, 282, 643

Muñoz Caro, G.M., Mennella, V., Ruiterkamp, R., Schutte, W.A., Greenberg, J.M., \& Ehrenfreund, P. 2000, to appear in Thermal Emission Spectroscopy and Analysis of Dust, Disks and Regoliths (workshop Houston 1999)

Pendleton, Y.J., Sandford, S.A., Allamandola, L.J., Tielens, A.G.G.M., \& Sellgren, K. 1994, ApJ, 437, 683

Schutte, W.A. 1988, The Evolution of Interstellar Organic Grain Mantles, PhD Thesis, Leiden

Tielens, A.G.G.M. 1999, in Formation and Evolution of Solids in Space, eds.

J.M. Greenberg \& A. Li (Dordrecht: Kluwer), 331 00, 1315

Wexler, A.S. 1967, Appl. Spectrosc. Rev., 1, 29 


\section{Discussion}

J.H. Black: If the PAH component of dust contributes much of the far-UV extinction, it will be purely absorbing. Can the net albedo in the far-UV be recovered with the other components?

J.M. Greenberg: We ( $\mathrm{Li} \&$ Greenberg 1997, A\&A, 323, 566 - a unified dust model) have calculated all properties for the complete mixture of core-mantle, hump particles and PAHs which gives the observed wavelength dependence of extinction and wavelength dependence of polarization. The net albedo is one of these properties and, within the error bars, matches the observed albedo.

U. G. Jørgensen: As I understand your dust model, it assumes the grain nucleus (core) to be silicates (the substrate in your experiments), and your experiments then simulate the grain growth. However, the interstellar grains identified in carbonaceous chondrites are mainly (i.e. almost only) carbon grains (diamonds). These probably represent the grain nucleus (the possible mantle may be destroyed in the laboratory in the chemical extraction process, or earlier). To what extent are your experiments sensitive to the use of a silicate substrate (rather than a carbon substrate)?

J.M. Greenberg: First, our experiments generally use an aluminium cold finger (substrate) in the cryostatic vacuum chamber. Second, once the surface is coated with a monolayer there is no further influence. The same holds for the interstellar dust silicate cores. With respect to interstellar diamonds, I would expect (observationally) that they are at least a minor component. As to how they were made, it is my impression that this is highly controversial. 